Pacific Journal of Mathematics

ENERGY BOUNDS AND VIRAL THEOREMS FOR ABSTRACT 


\title{
ENERGY BOUNDS AND VIRIAL THEOREMS FOR ABSTRACT WAVE EQUATIONS
}

\author{
L. E. Bobisud AND James Calvert
}

\begin{abstract}
The abstract wave equation $u^{\prime \prime}=A^{2} u+f(t, u)$ is considered on a Banach or Hilbert space, where $A$ generates a $\left(C_{0}\right)$ group. Under suitable conditions on $f$, a representation of the solution of the initial-value problem is used to establish bounds on the growth of the energy $1 / 2\|A u(t)\|^{2}+1 / 2\left\|u^{\prime}(t)\right\|^{2}$. For $f \equiv 0$ it is shown that neither the potential energy $1 / 2\|A u(t)\|^{2}$ nor the kinetic energy $1 / 2\left\|u^{\prime}(t)\right\|^{2}$ tends to zero as $t \rightarrow \infty$, and necessary and sufficient conditions for the kinetic and potential energies to be equal for large time are given.
\end{abstract}

Hille [8] has shown that the Cauchy problem for the abstract wave equation $u^{\prime \prime}=A^{2} u$ on a Banach space $\mathfrak{X}$ is well posed if and only if $A$ generates a strongly continuous group; hence we shall suppose throughout that $A$ is the generator of such a group. Hersh [7] has given a representation theorem for solutions of abstract initial-value problems in terms of distributional solutions of the Cauchy problem for certain related partial differential equations in two variables-in our case, in terms of solutions of $U_{t t}=U_{x x}$. Here we shall exploit his simple, explicit formula for the solution of the Cauchy problem for $u^{\prime}=A^{2} u+f$ to establish rather easily some results concerning the energy of the solution.

In the first part we establish estimates on the growth of the energy of a solution to the nonhomogeneous problem. The results here complement and extend some results in [5] and [6].

In part two we consider only the homogeneous linear problem, and show first that neither the kinetic nor the potential energy can tend to zero as time increases. We then specialize to Hilbert space, where we establish a necessary and sufficient condition for the kinetic and potential energies to be equal and constant for large $t$ and data in some subspace; a similar result may be found in [4]. By utilizing a consequence of an abstract formulation of Huyghens' principle, we are able to establish very easily a theorem of Duffin [2] on the equality of kinetic and potential energies of solutions of the three-dimensional wave equation with compactly supported data.

I. Energy bounds for abstract wave equations. We shall consider first the energy $1 / 2\|A u(t)\|^{2}+1 / 2\left\|u^{\prime}(t)\right\|^{2}$ for the abstract wave equation

$$
u^{\prime \prime}=A^{2} u+f(t), \quad u(0)=u_{0} \in D\left(A^{2}\right), \quad u^{\prime}(0)=u_{1} \in D(A)
$$


on a Banach space $\mathfrak{X}$. We assume throughout that $f(t)$ is strongly continuous from $[0, \infty)$ to $D(A)$ and that $A f(t)$ is strongly continuous; instead, we cound assume that $f$ is strongly continuously differentiable [5, II. 2]. The first part of Theorems 1 and 2 is proved differently in [5] for $A$ invertible and $f \equiv 0$.

THEOREM 1. Let A generate the $\left(C_{0}\right)$ group $T(t)$ satisfying $\|T(t)\| \leqq$ $M$, and let $\int_{0}^{\infty}\|f(t)\| d t<\infty$. Then $u$ has bounded energy; specifically,

$$
\begin{aligned}
\frac{1}{\sqrt{6} M}\left\{\left\|A u_{0}\right\|^{2}+\left\|u_{1}\right\|^{2}\right. & \left.-\left[\left.\sqrt{6} M\right|_{0} ^{\infty}\|f(t)\| d t\right]^{2}\right\}^{1 / 2} \\
& \leqq\left\{\|A u(t)\|^{2}+\left\|u^{\prime}(t)\right\|^{2}\right\}^{1 / 2} \\
& \leqq \sqrt{6} M\left\{\left\|A u_{0}\right\|^{2}+\left\|u_{1}\right\|^{2}+\left[\int_{0}^{\infty}\|f(t)\| d t\right]^{2}\right\}^{1 / 2} .
\end{aligned}
$$

Conversely, let $A$ generate a $\left(C_{0}\right)$ group $T(t)$, and suppose that for zero initial data the energy is bounded above by a nondecreasing function of $\int_{0}^{\infty}\|f(t)\| d t$. Then $T(t)$ is uniformly bounded.

Proof. By substitution one checks that the solution of (1) is given by

$$
\begin{aligned}
u(t)= & \frac{1}{2}[T(t)+T(-t)] u_{0} \\
& +\frac{1}{2} \int_{-t}^{t} T(s) u_{1} d s+\frac{1}{2} \int_{0}^{t} \int_{-(t-\tau)}^{t-\tau} T(s) f(\tau) d s d \tau ;
\end{aligned}
$$

this is the solution obtained in [7]. Since $A$ is closed and $d / d t T(t) v=$ $A T(t) v=T(t) A v$ for $v \in D(A)$, we obtain

$$
\begin{aligned}
A u(t)=\frac{1}{2}[T(t) & +T(-t)] A u_{0}+\frac{1}{2}[T(t)-T(-t)] u_{1} \\
& +\frac{1}{2} \int_{0}^{t}[T(t-\tau)-T(-(t-\tau))] f(\tau) d \tau, \\
u^{\prime}(t)= & \frac{1}{2}[T(t)-T(-t)] A u_{0}+\frac{1}{2}[T(t)+T(-t)] u_{1} \\
& +\frac{1}{2} \int_{0}^{t}[T(t-\tau)+T(-(t-\tau))] f(\tau) d \tau .
\end{aligned}
$$

Taking norms yield the upper bound in the first part of the theorem. For the lower bound, one obtains by adding and subtracting (3) and (4) and appropriate applications of $T(t)$ and $T(-t)$ that 


$$
\begin{aligned}
A u_{0}=\frac{1}{2}[T(t)+T(-t)] A u(t) & -\frac{1}{2}[T(t)-T(-t)] u^{\prime}(t) \\
& +\frac{1}{2} \int_{0}^{t}[T(\tau)-T(-\tau)] f(\tau) d \tau, \\
u_{1}=-\frac{1}{2}[T(t)-T(-t)] A u(t) & +\frac{1}{2}[T(t)+T(-t)] u^{\prime}(t) \\
& -\frac{1}{2} \int_{0}^{t}[T(\tau)-T(-\tau)] f(\tau) d \tau,
\end{aligned}
$$

whence the lower bound follows by taking norms.

For the converse, observe from (3) and (4) and the postulated energy bound that

$$
\left\|\int_{0}^{t} T(t-\tau) f(\tau) d \tau\right\| \leqq C\left(\int_{0}^{\infty}\|f(\tau)\| d \tau\right)
$$

where $C$ is a nondecreasing function of its argument. Let $\left\{\delta_{n}(t)\right\}$ be a sequence of nonnegative $C_{0}^{\infty}(-1,1)$ functions converging as $n \rightarrow \infty$ to the Dirac $\delta$-function; let $g \in D(A)$. Then

$$
\int_{0}^{t} T(t-\tau) \delta_{n}(\tau-\bar{t}) g d \tau \longrightarrow T(t-\bar{t}) g
$$

as $n \rightarrow \infty$ for $t>\bar{t}+1$. Also

$$
\int_{0}^{\infty} \delta_{n}(\tau-\bar{t})\|g\| d \tau=\|g\|,
$$

so (5) implies that

$$
\|T(s) g\| \leqq C(\|g\|)
$$

for $s \geqq 1$ and $g \in D(A)$. Since $A$ generates the $\left(C_{0}\right)$ group $T(t)$, we have that $\|T(t)\| \leqq M e^{\omega|t|}$ for some constants $M>0, \omega \geqq 0$. Thus $\|T(t) g\| \leqq \max \left(C(\|g\|), M e^{\omega}\right) \equiv K(\|g\|)$ for $t \geqq 0 ; K$ is nondecreasing.

Let $h \in \mathfrak{X}$ and $t \in(-\infty, \infty)$ be given. Let

$$
\varepsilon=\min \left(\|h\|, e^{-\omega t} K(2\|h\|) / M\right),
$$

and choose $g \in D(A)(\overline{D(A})=\mathfrak{X})$ such that $\|g-h\|<\varepsilon$. Then

$$
\begin{aligned}
\|T(t) h\| & \leqq\|T(t)(g-h)\|+\|T(t) g\| \\
& \leqq \varepsilon M e^{\omega t}+K(\|g\|) \leqq 2 K(2\|h\|), \quad t \geqq 0 .
\end{aligned}
$$

A similar argument works for $t \leqq 0$. Thus the family $\{T(t):-\infty<$ $t<\infty\}$ is bounded on each $h \in \mathfrak{X}$, and hence $\sup _{-\infty<t<\infty}\|T(t)\|<\infty$ by the uniform boundedness theorem.

Theorem 2. Let A generate a $\left(C_{0}\right)$ group $T(t)$ satisfying $\|T(t)\| \leqq$ 
$M e^{\omega ; t \mid}, \omega \geqq 0$, and suppose $\|f(t)\| \leqq \bar{M} e^{\gamma t}$ for $\gamma \geqq 0, t>0$; then

$$
\begin{aligned}
{\left[\|A u(t)\|^{2}+\left\|u^{\prime}(t)\right\|^{2}\right]^{1 / 2} } & \leqq C_{1} e^{\omega t}\left\{\left\|A u_{0}\right\|+\left\|u_{1}\right\|\right\} \\
& +C_{2} \int_{0}^{t} e^{\omega(t-\tau)}\|f(\tau)\| d \tau \\
& \leqq C_{3}\left(u_{0}, u_{1}, f\right) \begin{cases}e^{t} \max (\gamma, \omega), & \omega \neq \gamma \\
(1+t) e^{\omega t}, & \omega=\gamma\end{cases}
\end{aligned}
$$

for certain constants $C_{1}, C_{2}$ independent of the data.

Conversely, let $A$ generate the $\left(C_{0}\right)$ group $T(t)$, and suppose that for zero initial data the energy inequality (6) is satisfied for all bounded strongly continuous $f \in D(A)$ with Af strongly continuous; then

$$
\|T(t)\| \leqq M e^{\omega|t|}
$$

for some constant $M$.

The proof is similar to that of Theorem 1 and will be omitted.

Small perturbations of the differential equation should cause small changes in the rate of growth of the energy. This is the content of the following theorem.

THEOREM 3. Let A generate the $\left(C_{0}\right)$ group $T(t)$ satisfying $\|T(t)\| \leqq$ $M e^{\omega|t|}(\omega \geqq 0)$, and let $f(t)$ satisfy $\|f(t)\| \leqq \bar{M} e^{\gamma t}(\gamma \geqq 0)$. Let $g: R^{+} \times$ $\mathfrak{X} \rightarrow \mathfrak{X}$ satisfy

$$
\|g(t, u)\| \leqq K\{\|A u\|+\|u\|\}
$$

for some constant $K$ and $u \in D(A)$, in addition to hypotheses which guarantee the existence of solutions on $[0, \infty)$ to the problem (see [5] for such conditions)

(8) $u^{\prime \prime}=A^{2} u+g(t, u)+f(t) \quad u(0)=u_{0} \in D\left(A^{2}\right), u^{\prime}(0)=u_{1} \in D(A)$.

Then for any $\varepsilon>0$ there exists $K_{0}$ such that if the constant $K$ in (7) satisfies $0<K<K_{0}$, the energy growth estimate

$$
\left\|u^{\prime}(t)\right\|+\|A u(t)\| \leqq \text { const. } e^{t(\max (\gamma, \omega)+\varepsilon)}
$$

is valid. Conversely, given $K>0,(9)$ is satisfied by the solution $u$ of (8) for any $\varepsilon>4 M K \max \left(1, \omega^{-1}\right)$.

REMARK. If $B$ is a closed operator with $D(B) \supset D(A)$, then $\|B u\| \leqq$ $K\{\|A u\|+\|u\|\}$ for some constant $K$ and $u \in D(A)$, and solutions of $u^{\prime \prime}=\left(A^{2}+B\right) u+f$ exist [5], so our theorem covers this case of linear perturbation. Also included is the case where $g(t, u)$ satisfies 
a Lipschitz condition in $u$ uniformly in $t$.

Proof. We shall treat only the case $\omega>0, \omega \neq \gamma$; the other cases are similar. The solution $u$ of (8) satisfies the integral equation

$$
\begin{aligned}
u(t)=\frac{1}{2}[T(t)+T(-t)] u_{0} & +\frac{1}{2} \int_{-t}^{t} T^{\prime}(s) u_{1} d s \\
& +\frac{1}{2} \int_{0}^{t} \int_{-(t-\tau)}^{t-\tau} T(s) f(\tau) d s d \tau \\
& +\frac{1}{2} \int_{0}^{t} \int_{-(t-\tau)}^{t-\tau} T(s) g(\tau, u(\tau)) d s d \tau,
\end{aligned}
$$

whence we get the estimates

$$
\begin{aligned}
&\|u(t)\| \leqq M e^{\omega t}\left[\left\|u_{0}\right\|\right.\left.+\frac{1}{\omega}\left\|u_{1}\right\|\right]+ \\
&+\frac{M \bar{M}}{\omega|\omega-\gamma|} e^{t \max (\gamma, \omega)} \\
&+\frac{M K}{\omega} \int_{0}^{t} e^{\omega(t-\tau)}\{\|A u(\tau)\|+\|u(\tau)\|\} d \tau, \\
&\|A u(t)\|,\left\|u^{\prime}(t)\right\| \leqq M e^{\omega t}\left[\left\|A u_{0}\right\|+\left\|u_{1}\right\|\right]+\frac{M \bar{M}}{|\omega-\gamma|} e^{t \max (\gamma, \omega)} \\
&+M K \int_{0}^{t} e^{\omega(t-\tau)}\{\|A u(\tau)\|+\|u(\tau)\|\} d \tau .
\end{aligned}
$$

Set $\sigma=\max (\gamma, \omega)+\varepsilon$ for convenience; then for the three quantities above we have the bounds

$$
\begin{aligned}
& \|u(t)\|,\|A u(t)\|,\left\|u^{\prime}(t)\right\| \\
\leqq & C e^{\sigma t}+M K \max \left(1, \omega^{-1}\right) \int_{0}^{t} e^{\omega(t-\tau)}\{\|A u(\tau)\|+\|u(\tau)\|\} d \tau
\end{aligned}
$$

for a constant $C$ depending on the data and $\gamma, \omega$. We insist that $K<\varepsilon / 4 M \max \left(1, \omega^{-1}\right)$ and define

$$
S=C\left[\frac{1}{2}-\frac{2 M K}{\varepsilon} \max \left(1, \omega^{-1}\right)\right]^{-1}
$$

Since $S>2 C$, we see from (11) that

$$
\|u(t)\|,\|A u(t)\|,\left\|u^{\prime}(t)\right\|<S e^{o t}
$$

holds for positive $t$ near zero. Suppose $\bar{t}$ is the first positive time such that one of the strict inequalities (12) fails. Thus (12) holds on $[0, \bar{t}$ ), whence (11) yields for $t \in[0, \bar{t})$

$$
\|u(t)\|,\|A u(t)\|,\left\|u^{\prime}(t)\right\| \leqq C e^{\sigma t}+\frac{1}{\varepsilon} 2 M K \max \left(1, \omega^{-1}\right) S e^{\sigma t}<\frac{1}{2} S e^{\sigma t} ;
$$

thus $\|u(\bar{t})\|,\|A u(\bar{t})\|,\left\|u^{\prime}(\bar{t})\right\| \leqq(1 / 2) S e^{\sigma \bar{t}}$ by continuity. But then (12) 
holds at $\bar{t}$. This contradiction proves that (12) must hold on $[0, \infty)$, establishing the theorem.

The following theorem sharpens a result of [6] in the case $\omega>0$, $\gamma>0$; it is, however, weaker if $\gamma=0$ or $\omega=0$.

THEOREM 4. Let A generate a $\left(C_{0}\right)$ group $T(t)$ satisfying $\|T(t)\| \leqq$ $M e^{\omega|t|}$, and let $B$ be a closed linear operator on $X$ such that $D(B) \supset$ $D(A)$; let $u$ be a strong solution of

$$
u^{\prime \prime}=\left(A^{2}+B\right) u+f, \quad u(0)=u_{0} \in D\left(A^{2}\right), \quad u^{\prime}(0)=u_{1} \in D(A),
$$

where $f$ satisfies $\|f(t)\| \leqq \bar{M} e^{r t}$. Suppose the estimates

$$
\|u(t)\|,\|A u(t)\| \leqq \text { const. } e^{(\omega+|\gamma-\omega|) t}
$$

are satisfied on $[0, \infty)$. Then for any $\varepsilon>0$

$$
\left\|u^{\prime}(t)\right\| \leqq \text { const. } e^{(\omega+|\gamma-\omega|+\varepsilon) t}
$$

holds for $t \geqq 0$.

Outline of proof: Set $C_{\alpha}=A-\alpha I$ for $\alpha=|\gamma-\omega|+\varepsilon$; then $u$ satisfies

$$
u^{\prime \prime}=C_{\alpha}^{2} u+\left[2 \alpha A+B-\alpha^{2} I\right) u+f .
$$

$C_{\alpha}$ generates the $\left(C_{0}\right)$ group $T_{\alpha}(t)=e^{-\alpha t} T(t)$, where $\left\|T_{\alpha}(t)\right\| \leqq M e^{\omega|t|} e^{-\alpha t}$. Also $B$ satisfies the estimate $\|B u\| \leqq K\{\|A u\|+\|u\|\}$ for some constant $K$ and $u \in D(A)$. After converting (13) to the integral equation

$$
\begin{aligned}
u(t) & =\frac{1}{2}[T(t)+T(-t)] u_{0}+\frac{1}{2} \int_{-t}^{t} T(s) u_{1} d s \\
& +\int_{0}^{t} \int_{-(t-\tau)}^{t-\tau} T(s)\left[f(\tau)+\left\{2 \alpha A+B-\alpha^{2} I\right\} u(\tau)\right] d s d \tau,
\end{aligned}
$$

standard estimates yield the result, as in the preceding proof.

II. Virial theorems for $u^{\prime \prime}=A^{2} u$. Studying the solutions of $u^{\prime \prime}=A^{2} u$ on a Hilbert space where $A$ generates a unitary group, Shinbrot [9] and Goldstein [3] have established conditions under which the potential energy $1 / 2\|A u(t)\|^{2}$ and the kinetic energy $1 / 2\left\|u^{\prime}(t)\right\|^{2}$ approach a common limit. This virial theorem fails for solutions of the one-dimensional wave equation on $[0,1]$ with zero boundary data, where the lim inf of both the kinetic and potential energy can be zero. A weaker, related result is true, however; namely, that neither the kinetic nor the potential energy can have limit zero as time increases.

THEOREM 5. Let $A$ generate a norm-preserving group $T(t)$ on the 
Banach space $\mathfrak{X}$. Let $u$ be the solution of

$$
u^{\prime \prime}=A^{2} u, \quad u(0)=u_{0}, \quad u^{\prime}(0)=u_{1},
$$

where $u_{0} \in D\left(A^{2}\right), u_{1} \in D(A)$, and $\left\|u_{0}\right\|+\left\|u_{1}\right\|>0$. Then $\left\|u^{\prime}(t)\right\| \nrightarrow 0$, $\|A u(t)\| \nrightarrow 0$ as $t \rightarrow \infty$ unless $u_{1}=0$ and $A^{2} u_{0}=0$.

Proof. Assume to the contrary that $u^{\prime}(t) \rightarrow 0$ for certain initial data $u_{0}, u_{1}$. From (4) we have that

$$
T(t)\left[A u_{0}+u_{1}\right]-T(-t)\left[A u_{0}-u_{1}\right] \longrightarrow 0,
$$

whence (3) implies that

$$
A u(t)-\left\{\begin{array}{l}
T(t) \quad\left[A u_{0}+u_{1}\right] \\
T(-t)\left[A u_{0}-u_{1}\right]
\end{array}\right\} \longrightarrow 0 .
$$

From (4) we also get

$$
\begin{aligned}
& 2\left\|u^{\prime}(t)\right\|=2\left\|T(t) u^{\prime}(t)\right\|=\left\|T(2 t)\left[A u_{0}+u_{1}\right]-\left[A u_{0}-u_{1}\right]\right\| \longrightarrow 0, \\
& 2\left\|u^{\prime}(t)\right\|=2\left\|T(-t) u^{\prime}(t)\right\|=\left\|T(-2 t)\left[A u_{0}-u_{1}\right]-\left[A u_{0}+u_{1}\right]\right\| \longrightarrow 0 ;
\end{aligned}
$$

from this it follows that $T(t)\left[A u_{0}+u_{1}\right] \longrightarrow\left[A u_{0}-u_{1}\right], T(-t)\left[A u_{0}-\right.$ $\left.u_{1}\right] \rightarrow\left[A u_{0}+u_{1}\right]$. Thus $A u(t)$ converges to both $A u_{0}+u_{1}$ and $A u_{0}-$ $u_{1}$, which is impossible unless $u_{1}=0$.

Suppose then that $u_{1}=0$. The argument above shows that $T(t) A u_{0} \rightarrow A u_{0}$, so we have that

$$
\begin{aligned}
\left\|T(h) A u_{0}-A u_{0}\right\| & =\left\|T(t+h) A u_{0}-T(t) A u_{0}\right\| \\
& \leqq\left\|T(t+h) A u_{0}-A u_{0}\right\|+\left\|A u_{0}-T(t) A u_{0}\right\| \longrightarrow 0
\end{aligned}
$$

as $t \longrightarrow \infty$. Thus $T(h) A u_{0}=A u_{0}$ for all $h$, and so

$$
A^{2} u_{0}=\lim _{h \rightarrow 0} \frac{T(h) A u_{0}-A u_{0}}{h}=0 .
$$

This proves the theorem, for a similar argument holds if $A u(t) \rightarrow 0$.

In the case $A$ a skew-adjoint operator on a Hilbert space, $A^{2} u_{0}=$ 0 implies that $A u_{0}=0$, and hence the total energy vanishes.

Huyghens' principle for the hyperbolic equation

$$
u_{t t}=L[u] \text {, }
$$

where $L$ is an elliptic operator in $n$ space variables, asserts that for compactly supported data the solution will be zero at any fixed point in space for all sufficiently large time [2]. This principle is known to be valid for $L=\Delta_{n}$, the $n$-dimensional Laplacian, for $n=2 m+3$, $m=0,1, \cdots$. We shall give an abstract Hilbert space formulation of Huyghens' principle in order to derive information about the group 
generated by a square root of the Laplacian.

Let $\mathscr{H}$ be a Hilbert space, and let $\left\{H_{j}\right\}_{j=0}^{\infty}$ be a sequence of linear submanifolds of $\mathscr{H}$ such that $H_{0} \subset H_{1} \subset H_{2} \cdots$. We shall say that Huyghens' principle is valid for the abstract wave equation (14) relative to $\left\{H_{j}\right\}$ provided:

1. $D(A) \cap H_{j}$ is dense in $H_{j}, j=0,1, \cdots$, and $A\left(D(A) \cap H_{j}\right) \subset H_{j}$

2. for each $j=0,1, \cdots$ there exists a $T_{j}$ such that $|t|>T_{j}$ implies $\left(u(t), h_{j}\right)=0$ for $h_{j} \in H_{j}$ if $u_{0} \in D\left(A^{2}\right) \cap H_{0}, u_{1} \in D(A) \cap H_{0}$.

Example. Let $\mathscr{H}=\mathscr{L}^{2}\left(R^{3}\right), A^{2}=\Delta_{3}, H_{j}=\mathscr{L}^{2}\left(B_{j}\right)$, where $B_{j}$ is the ball of radius $C(j+1)$ for some constant $C>0$. Define the Fourier transform by

$$
F\{f\}(\xi)=\int e^{-i(x, \xi)} f(x) d x,
$$

where $x=\left(x_{1}, x_{2}, x_{3}\right), \xi=\left(\xi_{1}, \xi_{2}, \xi_{3}\right)$, and $(x, \xi)=x_{1} \xi_{1}+x_{2} \xi_{2}+x_{3} \xi_{3}$. Then we can define

$$
\begin{aligned}
A f & =i F^{-1}\left\{\left(\xi_{1}^{2}+\xi_{2}^{2}+\xi_{3}^{2}\right)^{1 / 2} F\{f\}\right\}, \\
D(A) & =\left\{f \in \mathscr{L}^{2}\left(R^{3}\right): f^{\prime} \text { exists and } f^{\prime} \in \mathscr{L}^{2}\left(R^{3}\right)\right\}
\end{aligned}
$$

it is easy to see that $A^{2}=\Delta_{3}$. A simple computation using the identity $\int F\{f\} g=\int f F\{g\}$ shows that $A$ is skew-adjoint and thus generates a $\left(C_{0}\right)$ unitary group on $\mathscr{H}$ (Stone's theorem). The validity of the abstract formulation of Huyghens' principle for the wave equation $u^{\prime \prime}=\Delta_{3} u$ relative to $\left\{H_{j}\right\}$ follows from the known classical result [1]. This example is readily generalized to $A^{2}=\Delta_{2 m+3}, m=0,1, \cdots$.

Theorem 6. Let $A$ generate a $\left(C_{0}\right)$ unitary group $T(t)$ on the Hilbert space $\mathscr{H}$, and let $\left\{H_{j}\right\}_{j=0}^{\infty}$ be an increasing sequence of linear manifolds in $\mathscr{H}_{\text {. Then }}\left(T(t) h_{0}, h_{j}\right)=0$ for $|t|>T_{j}$ and all $h_{0} \in H_{0}$, $h_{j} \in H_{j}$ if Huyghens' principle is valid for (14) relative to $\left\{H_{j}\right\}$.

Conversely, suppose $D(A) \cap H_{j}$ is dense in $H_{j}$ for $j=0,1, \cdots$, and there exist $T_{j}$ such that $|t|>T_{j}$ implies $\left(T(t) h_{0}, h_{j}\right)=0$ for each $h_{0} \in$ $H_{0}, h_{j} \in H_{j}$. Then $\left(u(t), h_{j}\right)=C_{j}$ for some constant $C_{j}$ (depending on the data for $u$ ) and all $h_{j} \in H_{j}$ if $u_{0} \in D\left(A^{2}\right) \cap H_{0}$ and $u_{1} \in D(A) \cap H_{0}$.

Proof. Let $h_{j}$ be an element of the dense set $D(A) \cap H_{j}$; then

$$
\begin{aligned}
-2\left(u(t), A h_{j}\right) & =2\left(A u(t), h_{j}\right) \\
& =\left(T(t)\left[A u_{0}+u_{1}\right]+T(-t)\left[A u_{0}-u_{1}\right], h_{j}\right)=0
\end{aligned}
$$

for $|t|>T_{j}$ since $A$ is skew-adjoint by Stone's theorem. Taking $u_{0}=$ 0 yields 


$$
\left(T(t) u_{1}, h_{j}\right)=\left(T(-t) u_{1}, h_{j}\right) .
$$

For $|t|>T_{j}$ we also have $(d / d t)\left(u(t), h_{j}\right)=\left(u^{\prime}(t), h_{j}\right)=0$, whence for $u_{0}=0$

$$
\left(T(t) u_{1}, h_{j}\right)=-\left(T(-t) u_{1}, h_{j}\right),
$$

so we must have $\left(T(t) u_{1}, h_{j}\right)=0$ for $|t|>T_{j}$ and all $u_{1} \in D(A) \cap H_{0}$, $h_{j} \in D(A) \cap H_{j}$. Since these sets are dense in $H_{0}$ and $H_{j}$ respectively, the first part of the theorem follows by a simple approximation argument.

For the partial converse, observe that $\left(T(t) h_{0}, h_{j}\right)=0$ for all $h_{0} \in$ $H_{0}, h_{j} \in H_{j}$, and $|t|>T_{j}$ implies that $(d / d t)\left(u(t), h_{j}\right)=0$ for $|t|>T_{j}$ and data in $H_{0}$.

The converse cannot in general be strengthened to conclude that $\left(u(t), h_{j}\right)=0$. To see this, let $A=(d / d x)$ on $\mathscr{L}^{2}(-\infty, \infty)$ and $H_{j}=$ $\mathscr{L}^{2}(-(j+1),(j+1)) . \quad A$ generates the group $T(t) f(x)=f(x+t)$, and Huyghens' principle is not valid (readily seen from the classical solution of d'Alembert). Nevertheless, for $w \in H_{0}$,

$$
\left(T(t) w, h_{j}\right)=\int_{-\infty}^{\infty} w(x+t) \overline{h_{j}(x)} d x=0
$$

for $|t|>j+2$.

Duffin [2] has recently established a virial theorem for solutions of the classical three-dimensional wave equation with compactly supported data; see also [4]. Using the Paley-Wiener theorem, Duffin shows that for sufficiently large time the kinetic energy $1 / 2\left\|u^{\prime}(t)\right\|^{2}$ and the potential energy $1 / 2\|A u(t)\|^{2}$ are constant and equal. We shall derive this result as a corollary to the following theorem for abstract wave equations; the result itself is similar to one of [4].

TheOREM 7. Let $A$ generate the unitary $\left(C_{0}\right)$ group $T(t)$ on the Hilbert space $\mathscr{H}$. Let $H$ be a subspace of $\mathscr{H}$ such that $D(A) \cap H$ is dense in $H$ and $A(D(A) \cap H) \subset H$. Then, for solution $u$ of (14) with arbitrary data $u_{0} \in D\left(A^{2}\right) \cap H, u_{1} \in D(A) \cap H$, the kinetic and potential energies will be equal for $t>S$ if and only if

$$
\operatorname{Re}\left(T(2 t) h, h^{\prime}\right)=0
$$

for all $h, h^{\prime} \in H$ and $t>S$.

Proof. From (3), (4), and the parallelogram law we have

$$
\begin{aligned}
\|A u(t)\|^{2}= & \frac{1}{4}\left\|A u_{0}+u_{1}\right\|^{2}+\frac{1}{4}\left\|A u_{0}-u_{1}\right\|^{2} \\
& +\frac{1}{2} \operatorname{Re}\left(T(t)\left[A u_{0}+u_{1}\right], T(-t)\left[A u_{0}-u_{1}\right]\right)
\end{aligned}
$$




$$
\begin{aligned}
= & \frac{1}{2}\left\|A u_{0}\right\|^{2}+\frac{1}{2}\left\|u_{1}\right\|^{2} \\
& +\frac{1}{2} \operatorname{Re}\left(T(t)\left[A u_{0}+u_{1}\right], T(-t)\left[A u_{0}-u_{1}\right]\right), \\
\left\|u^{\prime}(t)\right\|^{2}= & \frac{1}{2}\left\|A u_{0}\right\|^{2}+\frac{1}{2}\left\|u_{1}\right\|^{2} \\
& -\frac{1}{2} \operatorname{Re}\left(T(t)\left[A u_{0}+u_{1}\right], T(-t)\left[A u_{0}-u_{1}\right]\right) ;
\end{aligned}
$$

thus the total energy $1 / 2\left\|u^{\prime}(t)\right\|^{2}+1 / 2\|A u(t)\|^{2}$ is constant for all time. Clearly, the kinetic and potential energies will be equal for $t>S$ for all allowable data in (14) if and only if $\operatorname{Re}\left(T(t)\left[A u_{0}+u_{1}\right], T(-t)\left[A u_{0}-\right.\right.$ $\left.\left.u_{1}\right]\right)=0$ for all $u_{0} \in D\left(A^{2}\right) \cap H$ and $u_{1} \in D(A) \cap H$. It is obvious that $\operatorname{Re}\left(T(2 t) h, h^{\prime}\right)=0$ for all $h, h^{\prime} \in H$ and $t>S$ implies that the kinetic energy equals the potential energy for $t>S$. Conversely, if the kinetic and potential energies are equal for $t>S$, then setting $u_{0}=0$ yields $\operatorname{Re}\left(T(2 t) u_{1}, u_{1}\right)=0$ for $t>S$ and all $u_{1} \in D(A) \cap H$. Since $D(A) \cap H$ is dense in $H$, an approximation argument guarantees that $\operatorname{Re}(T(2 t) h$, $h)=0$ for $t>S$ and $h \in H$. The polarization identity for the sesquilinear form $\varphi\left(h, h^{\prime}\right)=\left(T(2 t) h, h^{\prime}\right)$ shows that this is equivalent to $\operatorname{Re}\left(T(2 t) h, h^{\prime}\right)=0$ for $t>S$ and $h, h^{\prime} \in H$.

ExAMPle. Let $\mathscr{H}=\mathscr{L}^{2}(-\infty, \infty), H=\mathscr{L}^{2}(-C, C), A=d / d x$, $T(t) f(x)=f(x+t)$; then $S=2 C$.

Corollary (Duffin). Let $\mathscr{H}=\mathscr{L}^{2}\left(R^{2 m+3}\right), H=\mathscr{L}^{2}(B)$ for a ball $B \subset R^{2 m+3}, A^{2}=\Delta_{2 m+3}, m=0,1, \cdots$. Then the kinetic and potential energies for the wave equation $u^{\prime \prime}=\Delta_{2 m+3} u, u(0)=u_{0} \in D\left(A^{2}\right) \cap H, u^{\prime}(0)=$ $u_{1} \in D(A) \cap H$ are equal for all sufficiently large time.

This follows from Theorem 6 and the example preceding it if we take $j=0$ in the theorem and $C=$ radius of the ball $B$ in the example.

The following theorem is similar to results of Shinbrot [9] and Goldstein [3]; a proof can be given readily along the lines of the proof of Theorem 7 .

THEOREM 8. Let the skew-adjoint operator $A$ on the Hilbert space $\mathscr{H}$ generate the group $T(t)$ and let $u(t)$ be the solution of (14) with $u_{0} \in D\left(A^{2}\right), u_{1} \in D(A)$. Then

$$
\lim _{|t| \rightarrow \infty}\|A u(t)\|^{2}=\lim _{|t| \rightarrow \infty}\left\|u^{\prime}(t)\right\|^{2}=\frac{1}{2}\left(\left\|A u_{0}\right\|^{2}+\left\|u_{1}\right\|^{2}\right)
$$




$$
\lim _{|t| \rightarrow \infty} \operatorname{Re}\left(T(t)\left[A u_{0}+u_{1}\right], T(-t)\left[A u_{0}-u_{1}\right]\right)=0
$$

\section{REFERENCES}

1. R. Courant and D. Hilbert, Methods of Mathematical Physics, Vol. II: Partial differential equations, New York, Interscience, 1962.

2. R. J. Duffin, Equipartition of energy in wave motion, J. Math. AnalAppl., 32 (1970), 386-391.

3. J. A. Goldstein, An asymptotic property of solutions of wave equations, Proc. Amer. Math. Soc., 23 (1969), 359-363.

4. - As asymptotic property of solutions of wave equations II, J. Math. Anal. Appl., 32 (1970), 392-399.

5. - Semigroups of Operators and Abstract Cauchy Problems, Tulane University Lecture Notes, 1970 .

6. - On the growth of solutions of inhomogeneous abstract wave equations, J. Math. Anal. Appl., 37 (1972), 650-654.

7. R. Hersh, Explicit solution of a class of higher-order abstract Cauchy problems, J. Differential Equations, 8 (1970), 570-579.

8. E. Hille, Une généralisation du probléme de Cauchy, Ann. de l'Inst. Fourier (Grenoble), IV, Année 1952 (1953), 31-48.

9. M. Shinbrot, Asymptotic behavior of solutions of abstract wave equations, Proc. Amer. Math. Soc., 19 (1968), 1403-1406.

Received May 12, 1972.

UNIVERSITY OF IDAHO 



\section{PACIFIC JOURNAL OF MATHEMATICS}

\section{EDITORS}

D. Gilbarg and J. Milgram

Stanford University

Stanford, California 94305

R. A. Beaumont

University of Washington

Seattle, Washington 98105
J. DUGUNDJI* Department of Mathematics

University of Southern California Los Angeles, California 90007

RICHARD ARENS

University of California

Los Angeles, California 90024

\section{ASSOCIATE EDITORS}

E. F. BeCKenbach

B. H. NeUMaNN

F. WOLF

K. Yoshida

\section{SUPPORTING INSTITUTIONS}

UNIVERSITY OF BRITISH COLUMBIA

UNIVERSITY OF SOUTHERN CALIFORNIA

CALIFORNIA INSTITUTE OF TECHNOLOGY

UNIVERSITY OF CALIFORNIA

MONTANA STATE UNIVERSITY

STANFORD UNIVERSITY

UNIVERSITY OF TOKYO

UNIVERSITY OF NEVADA

UNIVERSITY OF UTAH

NEW MEXICO STATE UNIVERSITY

WASHINGTON STATE UNIVERSITY

OREGON STATE UNIVERSITY

UNIVERSITY OF OREGON

OSAKA UNIVERSITY

UNIVERSITY OF WASHINGTON

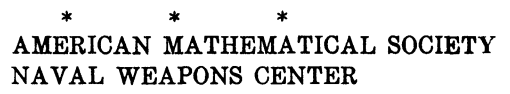

* C. DePrima will replace J. Dugundji until August 1974. 


\section{Pacific Journal of Mathematics}

\section{Vol. 47, No. $1 \quad$ January, 1973}

K. Adachi, Masuo Suzuki and M. Yoshida, Continuation of holomorphic

mappings, with values in a complex Lie group ....................

Michael Aschbacher, A characterization of the unitary and symplectic groups

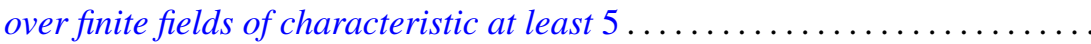

Larry Eugene Bobisud and James Calvert, Energy bounds and virial theorems for abstract wave equations....................................

Christer Borell, A note on an inequality for rearrangements ................

Peter Southcott Bullen and S. N. Mukhopadhyay, Peano derivatives and general

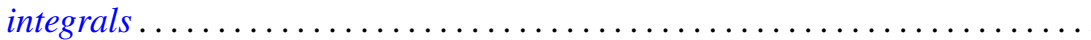

Wendell Dan Curtis, Yu-Lee Lee and Forrest Miller, A class of infinite dimensional subgroups of $\operatorname{Diff}^{r}(X)$ which are Banach Lie groups .........

Paul C. Eklof, The structure of ultraproducts of abelian groups ...............

William Alan Feldman, Axioms of countability and the algebra $C(X) \ldots \ldots \ldots$

Jack Tilden Goodykoontz, Jr., Aposyndetic properties of hyperspaces...........

George Grätzer and J. Płonka, On the number of polynomials of an idempotent algebra. II ...........................................

Alan Trinler Huckleberry, The weak envelope of holomorphy for algebras of

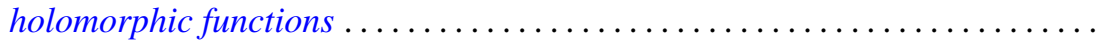

John Joseph Hutchinson and Julius Martin Zelmanowitz, Subdirect sum decompositions of endomorphism rings . . . . . . . . . . . . . . . .

Gary Douglas Jones, An asymptotic property of solutions of

$y^{\prime \prime \prime}+p y^{\prime}+q y=0$.

Howard E. Lacey, On the classification of Lindenstrauss spaces .

Charles Dwight Lahr, Approximate identities for convolution measure algebras.

George William Luna, Subdifferentials of convex functions on Banach

spaces.

Nelson Groh Markley, Locally circular minimal sets. .

Robert Wilmer Miller, Endomorphism rings of finitely generated projective modules

Donald Steven Passman, On the semisimplicity of group rings of linear

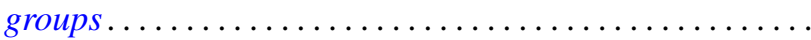

Bennie Jake Pearson, Dendritic compactifications of certain dendritic spaces.

Ryōtarō Satō, Abel-ergodic theorems for subsequences ...... .

Henry S. Sharp, Jr., Locally complete graphs. . .

Harris Samuel Shultz, A very weak topology for the Mikusinski field of

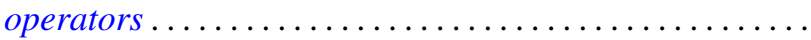

Elena Stroescu, Isometric dilations of contractions on Banach spaces ...

Charles W. Trigg, Versum sequences in the binary system ... . .

William L. Voxman, On the countable union of cellular decompositions of n-manifolds 